\title{
Performance Comparison of Various Image Denoising Filters Under Spatial Domain
}

\author{
Inderpreet Singh \\ M. Tech Student \\ Department of Computer Engineering \\ Punjabi University, Patiala
}

\author{
Nirvair Neeru \\ Assistant Professor \\ Department of Computer Engineering \\ Punjab University, Patiala
}

\begin{abstract}
Image denoising is very important during enhancement of image. Original Image is generally corrupted with various types of noise. The noise present in the images may appear as additive or multiplicative components. The most challenging problem is removing that noise from an Image while preserving its details. Several noise removal techniques have been developed so far each having its own advantages and disadvantages. The focus of this paper is to study various spatial filters and to compare their performance in removing different types of noise. Here quantitative measure of comparison is provided by the Peak Signal to Noise Ratio (PSNR) parameter.
\end{abstract}

\section{General Terms}

Image Denoising, Spatial filtering.

\section{Keywords}

Image denoising, Additive or Multiplicative Noise, Peak Signal to Noise Ratio.

\section{INTRODUCTION}

Digital images play an crucial role in different areas like television, remote sensing, ultrasound, CT scan etc. They are also used in various research areas like Uranology. Images captured by different devices generally adds the different types of noise in them while capturing due to faulty instruments or wrong methods of data capturing. Sometimes noise is added to an image during its transmission over various media. So, denoising the image is an essential task and it is generally done before considering the image for various purpose. An Ideal denoising technique should be able to remove most of noise from image while preserving its fine details [17].

Image denoising is considered as an important step and is generally done prior to processing of an image. It shows the process of recovering a good estimate of the original image from a corrupted image without modifying the useful structure in the image such as edges, discountinuities and fine details [9]. Generally speaking, denoising is the process of removing the unwanted noise from the corrupted image and reconstructing the original image. The main challenge is to design such noise removing techniques which should be able to remove most of noise from noisy image with minimum or no loss of its significant details [13]. It has many applications in other domains like object recognition, digital entertainment, and remote sensing imaging etc. As the number of image sensors per unit area increases, camera devices capture the noise with the image more often. Denoising techniques have become a vital step for improving the visual quality of images which are degarded by different types of noise [2] [6] [7].

Noise can be categorized as Gaussian noise, Uniform noise, Impulse noise (salt and pepper noise)[14] [12] Erlang noise
/Gamma noise, Rayleigh noise and Speckle noise each having its own probability density function.

This paper is organized as follows. In section 2 noise model for different types of noise are defined. Section 3 gives the various Spatial image denoising techniques. Section 4 gives the implementation of various filters on images corrupted with different types of noise. Finally, Section 5 gives the conclusion and Section 6 gives the Furture scope of the work. At the end, Appendix is given which consists of 4 Tables and 4 Figures which shows the performance of various Spatial filters.

\section{NOISE MODELS}

Noise is generally added to image during image capturing or due to faulty image capturing hardware. For e.g. during acquiring images with CCD camera, the two major factors which affect the amount of noise in the image are sensor temperature and light levels. Images are also corrupted during transmission due to interference in the channel [11]

The degradation process is shown below. Here degradation function and additive noise, both are added to the original input image $\mathrm{f}(\mathrm{x}, \mathrm{y})$ to produce a degraded image $\mathrm{g}(\mathrm{x}, \mathrm{y})$. Given $\mathrm{g}(\mathrm{x}, \mathrm{y})$, some idea about the degradation function $\mathrm{H}$ and additive noise term $n(x . y)$, one can acheive the estimate $f^{\wedge}$ (x.y), of the original input image by using the restoration model. In general, the more one has idea about $\mathrm{H}$ and $\mathrm{n}(\mathrm{x}, \mathrm{y})$, the closer estimate to $\mathrm{f}(\mathrm{x}, \mathrm{y})$ one will obtain. The degradation model can be represented with the following equation.

$g(x, y)=h(x, y) \times f(x, y)+n(x, y)$

Here $\mathrm{f}(\mathrm{x}, \mathrm{y})$ is the original image pixel value and $\mathrm{n}(\mathrm{x}, \mathrm{y})$ is the additive noise, $\mathrm{h}(\mathrm{x}, \mathrm{y})$ be the degradation function and $\mathrm{g}(\mathrm{x}, \mathrm{y})$ is the resulting noise image. [19]

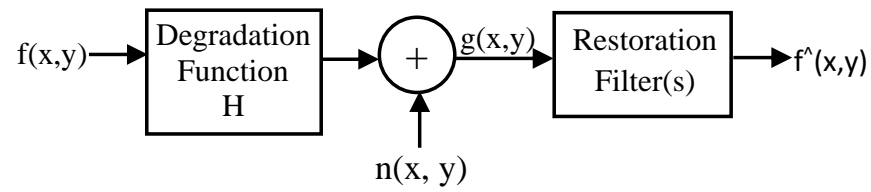

Fig. 1 A model of the image degradation/restoration process [19]

The Different types of Noise models are described below :

\subsection{Gaussian Noise or Amplifier Noise}

It is also known as Gaussian distribution. It has a probability density function (PDF) of the normal distribution. This noise is added to image during image acquisition like sensor noise caused by low light, high temperature, transmission e.g. electronic circuit noise [7]. This noise can be removed by using spatial filtering (mean filtering, median filtering and gaussian smoothing) by smoothing the image but smoothing also blurs the fine-scaled image edges and details. [4]. The PDF of Gaussian Noise is shown in the following equation and figure : 


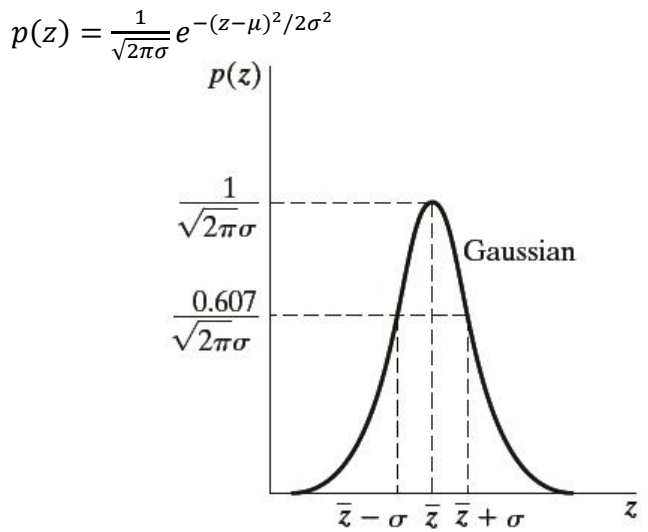

Fig. 2 PDF of Gaussian Noise

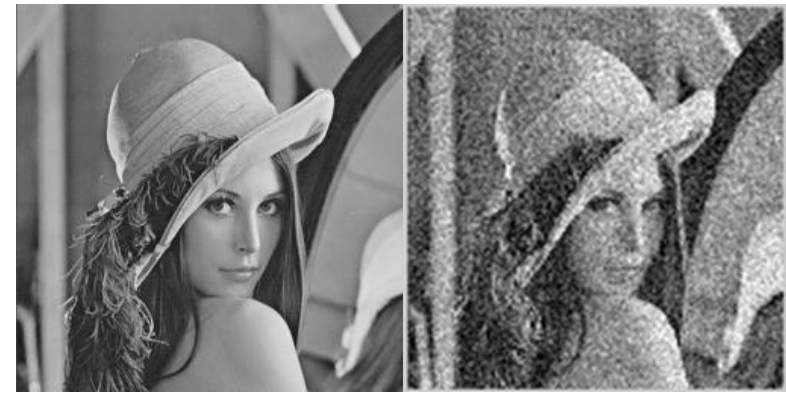

Fig. 3 Image containing Gaussian Noise

\subsection{Impulse Noise}

The Impulse noise is also known as Salt \& Pepper noise or Spike noise. It is caused by malfunctioning pixels in camera sensors, faulty memory locations in hardware, or transmission in a noisy channel [1]. It is always Independent and uncorrelated to image pixels. Its two types are the salt-andpepper noise and the random-valued noise. In salt and pepper type of noise, the noisy pixels takes either salt value (gray level -225) or pepper value (grey level -0) and it appears as black and white spots on the images In case of random valued impulse noise, noise can take any gray level value from zero to 225 . In this case also noise is randomly distributed over the entire image and probability of occurrence of any gray level value as noise will be same [5].

\section{Reasons for Salt and Pepper Noise:}

1) Due to failure of memory cells or wrong working of sensor cells of camera.

2) Due to synchronization errors while transmitting image over media [18].

The PDF of Impulse noise is shown in following equation and figure :

$$
\mathrm{p}(\mathrm{z})= \begin{cases}p_{a} & \text { for } z=a \\ p_{b} & \text { for } z=b \\ 0 & \text { otherwise }\end{cases}
$$

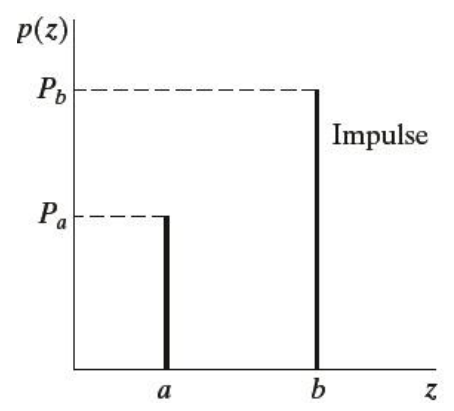

Fig. 4 PDF of Impulse (Salt \& pepper) Noise

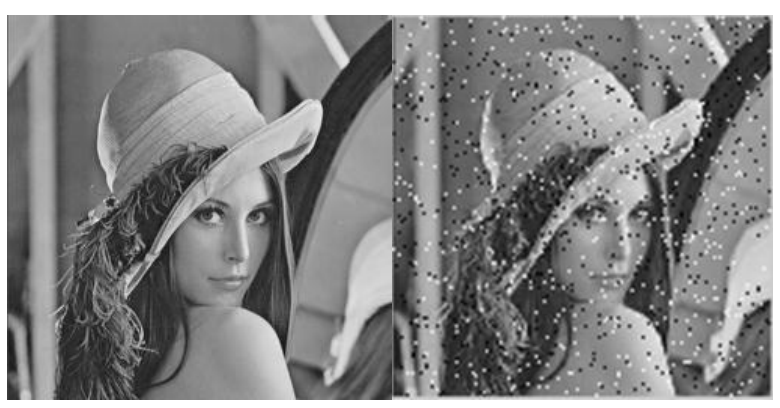

Fig. 5 Image containing Impulse Noise

\subsection{Speckle Noise}

Speckle noise is a granular noise. This noise generally degrades Synthetic Aperture Radar (SAR) images to large extent. This noise is generally caused due to random ups and downs in the signal coming back from an object that is smaller than a single image-processing element. It is also caused by consistent processing of backscattered signals from a no of distributed targets. This noise also increases the mean grey level of affecting image. This noise creates a lot of difficulty in interpreting the image. [20].

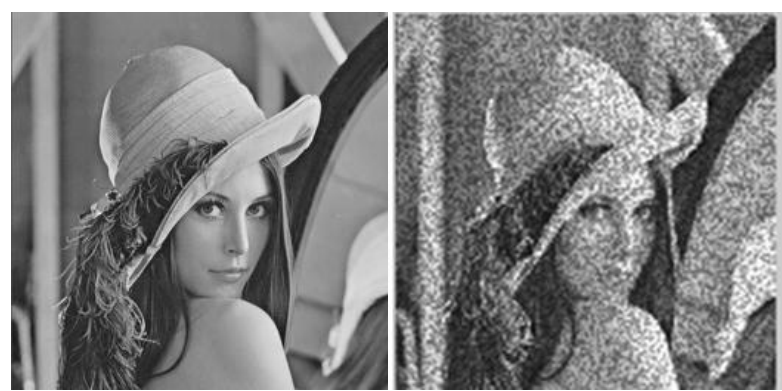

Fig. 6 Image Containing Speckle Noise

\subsection{Poisson Noise}

Poisson noise is also known as Photon noise. It arises when number of photons sensed by the sensor is not sufficient to provide detectable statistical information [16]. This noise has root mean square value proportional to square root intensity of the image. Different pixels are suffered by independent noise values. The photon noise and other sensor based noise corrupt the signal at different proportions [15]. The PDF of Poisson Noise is shown in following equation and figure :

$p(x)=\frac{e^{-\lambda} \lambda^{x}}{x !}$ for $\lambda>0$ and $\mathrm{x}=0,1,2 \ldots$

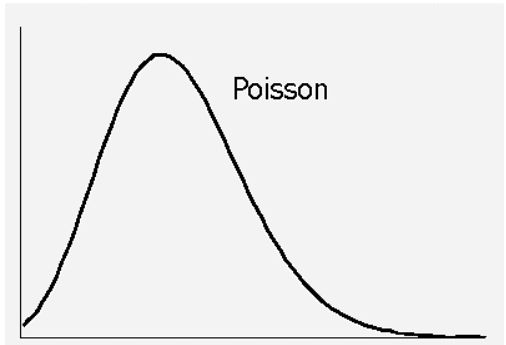

Fig. 7 PDF of Poisson Noise 


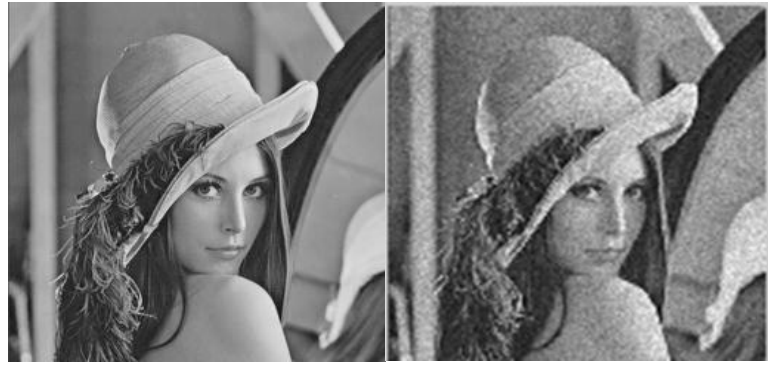

Fig. 8 Image containing Poisson Noise

\subsection{Uniform Noise}

The Uniform noise caused by quantizing the pixels of image to a number of distinct levels is known as Quantization noise. It has approximately uniform distribution. In this type of noise, the level of the gray values of the noise are uniformly distributed over a specified range. It can be used to create any type of noise distribution. This type of noise is mostly used to evaluate the performance of image restoration algorithms. This noise provides the most neutral or unbiased noise [10]. The PDF, mean and variance of Uniform Noise is shown below:

$$
p(z)=\left\{\begin{array}{c}
\frac{1}{(b-a)}, \text { if } a \leq z \leq b \\
0, \text { otherwise }
\end{array}\right.
$$

$\mu=(a+b) / 2$

$\sigma^{2}=(b-a)^{2} / 12$

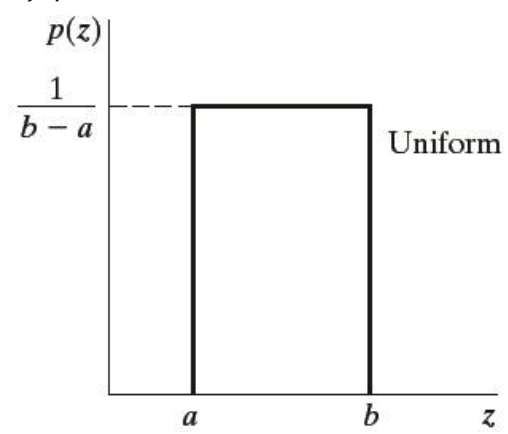

Fig. 9 PDF of uniform noise

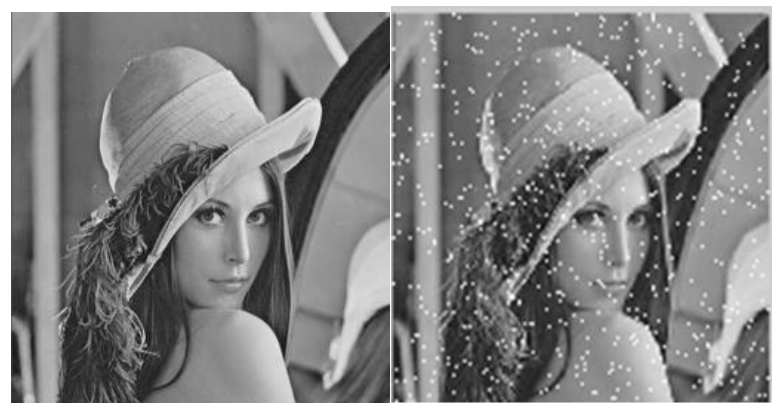

Fig. 10 Uniform Noise present in an Image

\subsection{Rayleigh Noise}

Radar range and velocity images typically contain noise that can be modelled by the Rayleigh distribution [18]. The PDF, mean and variance of Rayleigh Noise is given below:

$$
p(z)= \begin{cases}\frac{2}{b}(z-a) e^{\frac{-(z-a)^{2}}{b}} & \text { for } z \geq a \\ 0 & \text { for } z<a\end{cases}
$$

$$
\begin{aligned}
& \mu=a+\sqrt{\pi b / 4} \\
& \sigma^{2}=\frac{b(4-\mu)}{4}
\end{aligned}
$$

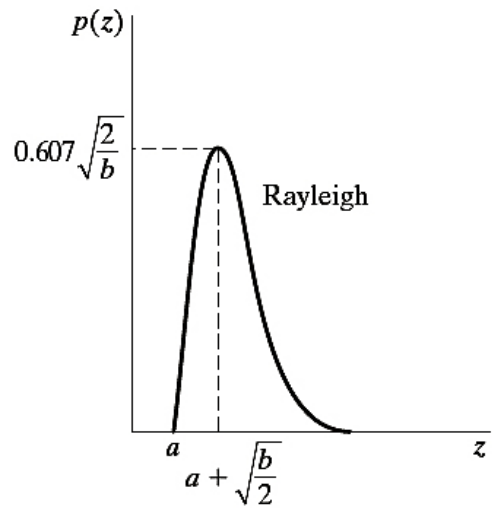

Fig. 11 PDF of Rayleigh Noise

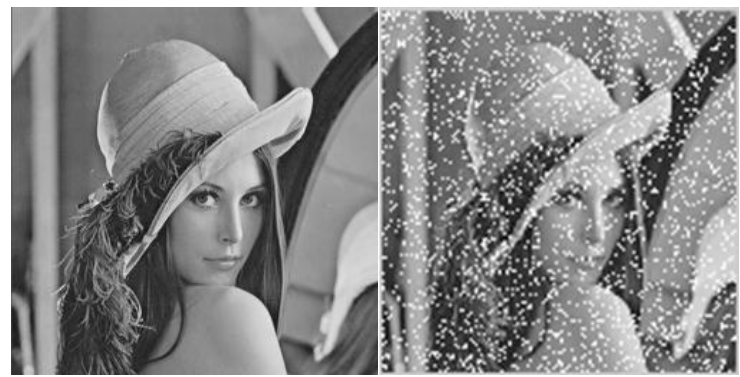

Fig. 12 Image containing Rayleigh Noise

\subsection{Gamma Noise}

This type of noise can be obtained by the low-pass filtering of laser based images [18]. The PDF, mean and variance of Gamma Noise is given below:

$f(x)= \begin{cases}\frac{a^{z} z^{b-1}}{(b-1) !} e^{-a z}, & \text { for } z<0 \\ 0, & \text { for } z \geq 0\end{cases}$

$\mu=b / a$

$\sigma^{2}=b / a^{2}$

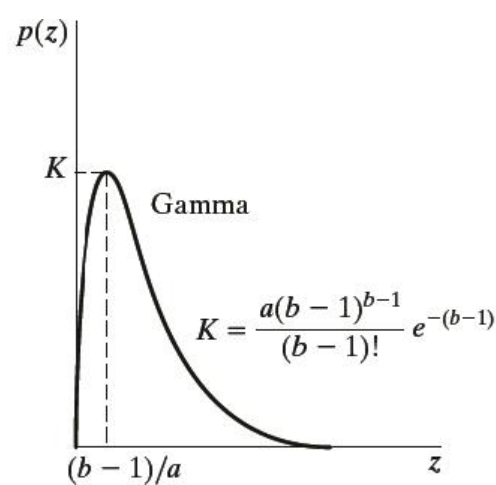

Fig. 13 PDF of Gamma noise 


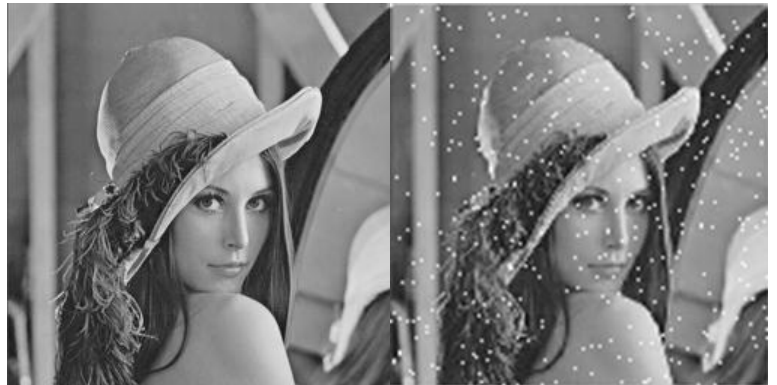

Fig. 14 Image containing Gamma Noise

\section{IMAGE DENOISING TECHNIQUES}

There are different Image denoising techniques developed so far each having its own advantages and limitation. One should choose the techniue accoring to the type and amount of noise present in the image. One should also consider the other factors like performance in denoising the image, computational time, computational cost.

Denoising can be done in various domains like Spatial Domain, Frequency Domain and Wavelet Domain. The Spatial domain method is discussed below.

\subsection{Spatial Domain}

Here filtering is used for image noise removal. Filtering is a technique in image processing which is used for different tasks like noise reduction, interpolation, and re-sampling. It is mostly used in all image processing systems. The choice of filter depend upon the type and amount of noise present in an image because different filters can remove different types of noise efficiently.

Spatial Domain has following types of filters :

\subsubsection{Linear Filters:}

Linear filters are used to remove certain type of noise. Here filtering is generally done by blurring the image. These filters blur the edges and destroy the fine details of an image. They have poor performance in removing signal dependent noise. Gaussian and Averaging filters are commonly used linear filters [8]. They are of following types :

\subsubsection{Gaussian Filter:}

Gaussian filter is a non-uniform low pass filter. Gaussian filter is used to blur images and remove noise and detail. It does not remove salt $\&$ pepper noise effectively [3].

\subsubsection{Average Filter:}

The output of average filter is simply the average of pixels contained in the neighborhood of filter mask. It calculates the average of all intensities of the neighbourhood of the central pixel and repacles the pixel with that average value. It is mostly used in removing irrelevant details from an image. It has a limitation that it blurs the edges of the image [19].

\subsubsection{Non-Linear Filters:}

In recent years, a variety of non-linear filters such as median filter, min filter, max filter have been developed to overcome the shortcoming of linear filter. Non-linear filters exhibit better performance than linear filters [10]. They are discussed below :

\subsubsection{Mean Filter:}

It is one of the most simplest filter among the existing spatial filters. It uses a filter window which is usually square. The filter window replaces the center value in the window with the average mean of all the pixels values in the kernel or window.

\subsubsection{Median Filter:}

It is also known as order statistics filter. It is most popular and commonly used non linear filter. It removes noise by smoothing the images. This filter also lowers the intensity variation between one and other pixels of an image. In this filter, the pixel value of image is replaced with the median value The median value is calculated by first arranging all the pixel values in ascending order and then replace the pixel being calcuated with the middle pixel value. If the neighbouring pixel of image which is to be consider, contains and even no of pixels, then it replaces the pixel with average of two middle pixel values. The median filter gives best result when the impulse noise percentage is less than 0.1 It does not perform well in removing high density salt \& pepper noise [19]. The mean filter can be represented by the following equation :

$f^{\wedge}(x, y)=\operatorname{median}\{g(s, t)\}$ where $(s, t) \in S_{x y}$

Here $S_{x y}$ corresponds to the set of coordinates in a rectangular subimage window which has center at $(\mathrm{x}, \mathrm{y})$. The median filter calculates the median of the corrupted image $g(x, y)$ under the area $S_{x y}$. Here $f^{\wedge}(x, y)$ represents the restored image.

\subsubsection{Min Filter:}

Min filter is also known as $0^{\text {th }}$ percentile filter. It replaces the value of pixel by the minimum intensity level of the neighborhood of that pixel.. This filter finds darkest points in an image. It removes salt noise from an image containing salt and pepper noise due to its high intensity value [19]. The min filter can be represented by the following equation :

$f^{\wedge}(x, y)=\min \{g(s, t)\}$ where $(s, t) \in S_{x y}$

\subsubsection{Max Filter:}

Max filter is also known as $100^{\text {th }}$ percentile filter. It replaces the value of pixel by the maximum intensity level of the neighborhood of that pixel. This filter finds brightest points in an image. It removes pepper noise from an image containing salt and pepper noise due to its very low intensity value [19].

$f^{\wedge}(x, y)=\max \{g(s, t)\}$ where $(s, t) \in S_{x y}$

\subsubsection{Adaptive Filters:}

These filters works accordingly the statistical characteristics of image inside inside the filter region defined by the $\mathrm{mxn}$ rectangular window. They are more complex and gives better performance than existing spatial filters. The most commonly used spatial filter is adaptive median filter which is discussed below :

\subsubsection{Adaptive Median Filter:}

It performs well on images containing high density salt \& pepper noise. It preserves the details of an image while smoothing non impulse noise. It changes its windows size during its operation depending on the certain conditions [19]. It works in two stages. First it calculates the minimum, maximum and median values of subimage window of the corrupted image. In stage one, it checks whether the calculated median itself is a salt or pepper noise or not. If the median is salt or pepper noise, then it increase the size of subimage window and recalculates the mimum, maximum and median values otherwise it proceeds to stage two. In stage two, it 
checks whether the selected pixel is a salt or pepper noise or not. If it is salt or pepper noise, then it replaces the selected pixel with previously calculated median otherwise the pixel remains unchanged.

\section{IMPLEMENTATION AND RESULTS}

Experiments were carried out on various standard grayscale images of size $256 \times 256$ which are of jpeg format and are shown in Figure 18. Simulation is performed using matlab R2013a software.
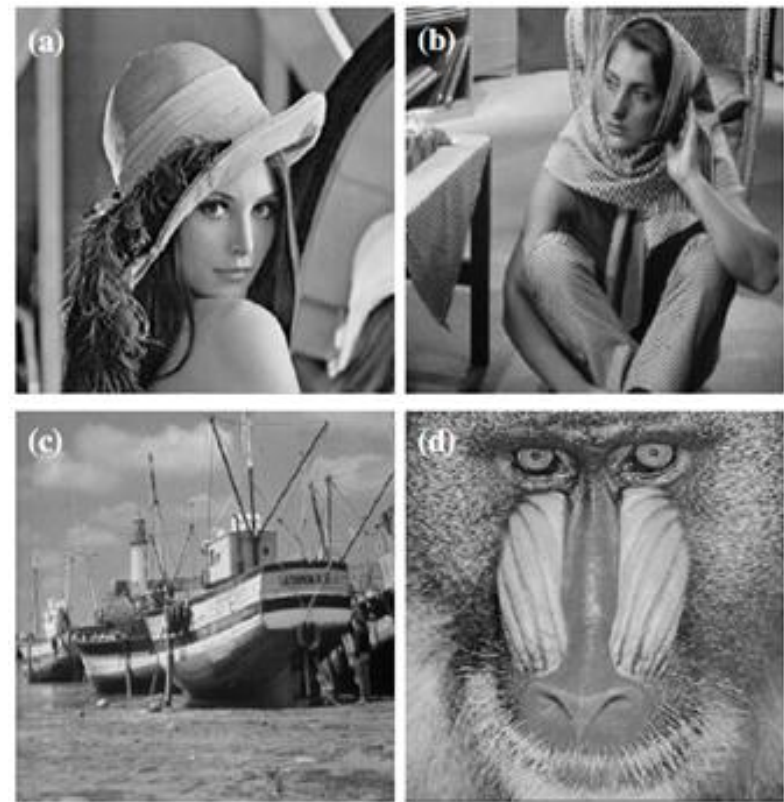

Fig. 15 Original Images used for simulation (a) Lena, (b) Barbara, (c) Boat, (d) Baboon

The input images are corrupted by a simulated Gaussian white noise (mean $=0$, variance $=0.01$ ), Salt \& Pepper noise (noise density $=0.05)$, Speckle noise $($ mean $=0$, variance $=0.04)$, Poisson noise, Uniform noise (interval [0,1]), Rayleigh noise (parameters 0,1), Erlang noise (parameters 2,5). For denoising process, various spatial linear filters which are gaussian filter (3x3), average filter $(3 \times 3)$ and spatial nonlinear filters which are median filter $(3 \times 3)$, min filter $(3 \times 3)$, max filter $(3 \times 3)$ and adaptive filters which are adaptive median filter $(3 \times 3)$, have been used.

The Quantitative performance of the spatial filters is evaluated through Peak signal to noise ratio (PSNR). It can be defined by following eq.

$P S N R=10 \log _{10}\left(\frac{255^{2}}{M S E}\right)$

$M S E=\frac{\sum_{i} \sum_{j}\left(r_{i j-} x_{i j}\right)}{M \times N}$

Where $r$ refers to Original image, $x$ denotes the restored image, $\mathrm{Mx} \mathrm{N}$ is the size of processed image.

Table 1 in the Appendix A shows performance of various spatial filters in removing the different types of noise in Lena image, in terms of psnr. Similarly Tables 2, 3 and 4 corresponds to Barbara, Boat and Baboon image respectively. Figure 16 shows the lena image corroupted with different noise types and each noisy image filtered using different filters. Similarly Figure 17, 18 and 19 corresponds to Barbara, Boat and Baboon image respectively.

\section{CONCLUSION}

In this paper, various noise models and filtering techniques like linear, nonlinear filtering and adaptive filtering have been discussed. The seven different types of noises which includes Gaussian noise, Salt \& Pepper noise, Speckle noise, Poisson noise, Uniform noise, Rayleigh noise and Erlang noise, were simulated on four different standard test images. Then six different spatial filters which includes Average filter, Gaussian filter, Min filter, Max filter, Median filter \& Adaptive Median filter, were applied on different noisy images.. The performance of the filters was evaluated using PSNR parameter. The comparison results show that Average filter shows better performance in removing Gaussian and Speckle noise while Gaussian filter removes Poisson noise efficiently. The adaptive median filters performed well in removing Salt \& Pepper, Uniform, Rayleigh and Erlang noise.

\section{FUTURE SCOPE}

This comparative study can be further extended by including more noise types like Exponential noise, Anisotropic noise, Film grain etc and/or by using multiple types of noise in different types of images. One can include more spatial filters using various means filters like Arithmetic mean filter, Geometric mean filter, Harmonic mean filter, Contraharmonic mean filter and order statistics filters like Midpoint filter, Alpha trimmed filter and Adative filters like Adaptive local noise reduction filter for comparison. One can also use hybrid filtering approach which involves two or more filters. Some other parameters like Entropy, Structure Similarity Index and Image Quality can also be considered for measuring the performance of different filters.

\section{REFERENCES}

[1] A. Bovik, Handbook of Image and Video Processing. New York: Academic, 2000.

[2] C. S. Lee, S. M. Guo, and C. Y. Hsu, "Genetic-based fuzzy image filter and its application to image processing," IEEE Trans. Syst. Man Cybern. B, bern., vol. 35, no. 4 pp. 694-711, Aug. 2005.

[3] Gaussian Noise [Online]. Available: https://www.cs.auckland.ac.nz/courses/Gaussian\%20Filt ering_lup.pdf

[4] Gaussian noise [Online]. Available: http://en.wikipedia.org/wiki/ Gaussian_noise

[5] J. Harikiran, B. Saichandana and B. Divakar, "Impulse Noise Removal in Digital Image." International Journal of Computer Applications, Vol. 10, no 8, pp. 39-42.

[6] J. H. Hong, S. B. Cho, and U. K. Cho, "A novel evolutionary approach to image enhancement filter design: method and applications," IEEE Trans. Syst. Man Cybern.B, bern., vol. 39, no. 6, pp. 1446-1457, Dec. 2009

[7] J. H. Wang, W. J. Liu, and L. D. Lin, "Histogram-based fuzzy filter for image restoration," IEEE Trans. Syst. Man Cybern. B, bern., vol. 32, no. 2, pp. 230-238, Apr. 2002.

[8] Keyur Patel and Hardik N. Mewada, "A Review on Different Image De-noising Methods", International Journal on Recent and Innovation Trends in Computing and Communication, Vol 2 Issue 1, 155-159 March 2014

[9] Kostadin Dabov, Alessandro Foi, Vladimir Katkovnik, and Karen Egiazarian, "Image denoising with blockmatching and 3D filtering" Image Processing: Algorithms and Systems, SPIE ,Electronic Imaging,Vol.6064,2006. 
[10] K. Somasundaram and P. Kalavathi, "Medical Image Denoising using Non-Linear Spatial Mean Filters for Edge Detection.”, rural.univ.ac.in, pp. 149-153

[11] K. S. Srinivasan, D. Ebenezer, "A New Fast and Efficient Decision-Based Algorithm for Removal of High-Density Impulse Noises", IEEE Signal Processing Letters, Vol. 14, No. 3, March 2007.

[12] Li Dan, Wang Yan and Fang Ting "Wavelet Image Denoising Algorithm based on Local Adaptive weiner filtering," International Conference on Mechatronic Science, Electrical Engineering and Computer August 1922, Jilin, China 2011.

[13] Ling Shao, Ruomei Yan and Xuelong Li, "From Heuristic Optimization to Dictionary Learning: A Review and Comprehensive Comparison of Image Denoising Algorithms," IEEE Transactions on Cybernetics, 1-14 August, 2013.

[14] Mehmet Sezgin and Bu" lent Sankur, "Survey on Image Thresholding Technique and quantitative performance evaluation", Journal of Electronic Imaging 13(1), 146165 January 2004.
[15] Mr. Amit Agrawal, Ramesh Raskar, "Optimal single image capture for motion deblurring", IEEE Conference on Computer Vision and Pattern Recognition, pages 25602567, 2009.

[16] Mr. Pawan Patidar and et al. Image De-noising by Various Filters for Different Noise in International Journal of Computer Applications (0975 - 8887) Volume 9- No.4, November 2010

[17] Mukesh C. Motwani, Mukesh C. Gadiya and Rakhi C. Motwani, "Survey of Image Denoising Techniques.," Proc. of GSPx, Santa Clara Convention Center, Santa Clara, CA, pp. 27-30, 2004.

[18] Priyanka Kamboj and Varsha Rani, "A Brief Study of Various Noise Model and filtering Techniques," Journal of Global Research in Computer Science, Volume 4, No 4, pp.166-171, April 2013.

[19] R. C. Gonzalez and R. E. Woods, "Digital Image Processing," second ed., Prentice Hall, Englewood, Englewood, Cliffs, NJ, 2002.

[20] Speckle noise [Online]. Available: http://en.wikipedia.org/wiki/Speckle_noise

\section{Appendix A}

Table 1. Peformance comparison of various filters on different types of noise using lena image

\begin{tabular}{|l|c|c|c|c|c|c|}
\hline \multirow{2}{*}{ Type of Noise } & \multicolumn{6}{|c|}{ Denoised Image PSNR } \\
\cline { 2 - 7 } & \multicolumn{2}{|c|}{ Linear Filters } & \multicolumn{2}{c|}{ Non Linear Filters } & $\begin{array}{c}\text { Adaptive } \\
\text { Filters }\end{array}$ \\
\cline { 2 - 7 } & $\begin{array}{c}\text { Gaussian } \\
\text { Filter }\end{array}$ & $\begin{array}{c}\text { Average } \\
\text { Filter }\end{array}$ & $\begin{array}{c}\text { Median } \\
\text { Filter }\end{array}$ & $\begin{array}{c}\text { Min } \\
\text { Filter }\end{array}$ & $\begin{array}{c}\text { Max } \\
\text { Filter }\end{array}$ & $\begin{array}{c}\text { Adaptive } \\
\text { Median Filter }\end{array}$ \\
\hline $\begin{array}{l}\text { Gaussian } \\
\text { noise }\end{array}$ & 23.7253 & 25.8026 & 25.4979 & 14.7697 & 14.6815 & 22.7467 \\
\hline $\begin{array}{l}\text { Salt \& } \\
\text { Pepper noise }\end{array}$ & 22.1794 & 24.9387 & 30.5088 & 12.1861 & 11.7244 & 37.4839 \\
\hline Speckle noise & 22.5712 & 25.1905 & 23.3750 & 14.7026 & 14.2945 & 20.7189 \\
\hline Poisson noise & 30.0853 & 27.5862 & 29.2124 & 18.8530 & 18.4931 & 28.7065 \\
\hline $\begin{array}{l}\text { Uniform } \\
\text { noise }\end{array}$ & 23.7290 & 25.4830 & 30.6693 & 20.8097 & 10.9641 & 34.6640 \\
\hline $\begin{array}{l}\text { Rayleigh } \\
\text { noise }\end{array}$ & 17.9421 & 20.7429 & 27.2467 & 20.9664 & 7.0116 & 29.3020 \\
\hline Erlang noise & 25.6125 & 26.3890 & 30.9288 & 20.7841 & 12.3587 & 34.9521 \\
\hline
\end{tabular}


Table 2. Performance comparison of various filters on different types of noise using barbara image

\begin{tabular}{|l|l|l|l|l|l|l|}
\hline \multirow{2}{*}{ Type of Noise } & \multicolumn{5}{|c|}{ Denoised Image PSNR } \\
\cline { 2 - 7 } & \multicolumn{2}{|c|}{ Linear Filters } & \multicolumn{2}{c|}{ Non Linear Filters } & \multicolumn{1}{c|}{$\begin{array}{c}\text { Adaptive } \\
\text { Filters }\end{array}$} \\
\cline { 2 - 7 } & $\begin{array}{c}\text { Gaussian } \\
\text { Filter }\end{array}$ & $\begin{array}{c}\text { Average } \\
\text { Filter }\end{array}$ & $\begin{array}{c}\text { Median } \\
\text { Filter }\end{array}$ & $\begin{array}{c}\text { Min } \\
\text { Filter }\end{array}$ & $\begin{array}{c}\text { Max } \\
\text { Filter }\end{array}$ & $\begin{array}{c}\text { Adaptive } \\
\text { Median Filter }\end{array}$ \\
\hline $\begin{array}{l}\text { Gaussian } \\
\text { noise }\end{array}$ & 23.5245 & 24.7801 & 24.2943 & 14.7023 & 14.4711 & 22.3013 \\
\hline $\begin{array}{l}\text { Salt \& } \\
\text { Pepper noise }\end{array}$ & 21.9990 & 24.0744 & 26.9224 & 12.7807 & 11.2108 & 30.6652 \\
\hline Speckle noise & 23.0974 & 24.6848 & 23.2255 & 15.1969 & 14.3174 & 21.1005 \\
\hline Poisson noise & 29.6819 & 26.1732 & 26.3985 & 18.7506 & 18.3279 & 27.3986 \\
\hline $\begin{array}{l}\text { Uniform } \\
\text { noise }\end{array}$ & 23.0142 & 24.3241 & 26.9576 & 20.4592 & 10.3217 & 30.7675 \\
\hline $\begin{array}{l}\text { Rayleigh } \\
\text { noise }\end{array}$ & 17.3943 & 20.0719 & 25.4286 & 20.6026 & 6.5150 & 28.1467 \\
\hline Erlang noise & 23.5245 & 24.7801 & 24.2943 & 14.7023 & 14.4711 & 22.3013 \\
\hline
\end{tabular}

Table 3. Peformance comparison of various filters on different types of noise using boat image

\begin{tabular}{|l|l|l|l|c|c|c|}
\hline \multirow{2}{*}{ Type of Noise } & \multicolumn{5}{|c|}{ Denoised Image PSNR } \\
\cline { 2 - 7 } & \multicolumn{2}{|c|}{ Linear Filters } & \multicolumn{2}{c|}{ Non Linear Filters } & \multicolumn{1}{c|}{$\begin{array}{c}\text { Adaptive } \\
\text { Filters }\end{array}$} \\
\cline { 2 - 7 } & $\begin{array}{c}\text { Gaussian } \\
\text { Filter }\end{array}$ & $\begin{array}{c}\text { Average } \\
\text { Filter }\end{array}$ & $\begin{array}{c}\text { Median } \\
\text { Filter }\end{array}$ & $\begin{array}{c}\text { Min } \\
\text { Filter }\end{array}$ & $\begin{array}{c}\text { Max } \\
\text { Filter }\end{array}$ & $\begin{array}{c}\text { Adaptive } \\
\text { Median Filter }\end{array}$ \\
\hline $\begin{array}{l}\text { Gaussian } \\
\text { noise }\end{array}$ & 23.7516 & 26.0948 & 25.7364 & 14.8648 & 14.6925 & 22.6735 \\
\hline $\begin{array}{l}\text { Salt \& } \\
\text { Pepper noise }\end{array}$ & 25.0062 & 25.0062 & 30.9745 & 11.7478 & 11.9887 & 35.7980 \\
\hline Speckle noise & 22.0179 & 25.1206 & 22.9734 & 14.3615 & 13.8254 & 20.1106 \\
\hline Poisson noise & 30.0707 & 27.9380 & 29.3486 & 18.9826 & 18.7393 & 28.5874 \\
\hline $\begin{array}{l}\text { Uniform } \\
\text { noise }\end{array}$ & 24.3334 & 26.0411 & 31.2354 & 21.2726 & 11.4607 & 36.0756 \\
\hline $\begin{array}{l}\text { Rayleigh } \\
\text { noise }\end{array}$ & 18.4553 & 21.2884 & 28.1451 & 21.4167 & 7.4662 & 30.6818 \\
\hline Erlang noise & 25.8548 & 26.7381 & 31.3529 & 21.2579 & 12.6767 & 36.2143 \\
\hline
\end{tabular}


Table 4. Performance comparison of various filters on different types of noise using baboor image

\begin{tabular}{|l|l|l|l|l|l|l|}
\hline \multirow{2}{*}{ Type of Noise } & \multicolumn{5}{|c|}{ Denoised Image PSNR } \\
\cline { 2 - 7 } & \multicolumn{2}{|c|}{ Linear Filters } & \multicolumn{2}{c|}{ Non Linear Filters } & \multicolumn{1}{c|}{$\begin{array}{c}\text { Adaptive } \\
\text { Filters }\end{array}$} \\
\cline { 2 - 7 } & $\begin{array}{c}\text { Gaussian } \\
\text { Filter }\end{array}$ & $\begin{array}{c}\text { Average } \\
\text { Filter }\end{array}$ & $\begin{array}{c}\text { Median } \\
\text { Filter }\end{array}$ & $\begin{array}{c}\text { Min } \\
\text { Filter }\end{array}$ & $\begin{array}{c}\text { Max } \\
\text { Filter }\end{array}$ & $\begin{array}{c}\text { Adaptive } \\
\text { Median Filter }\end{array}$ \\
\hline $\begin{array}{l}\text { Gaussian } \\
\text { noise }\end{array}$ & 23.3947 & 23.2433 & 22.8770 & 13.7440 & 13.6688 & 21.7374 \\
\hline $\begin{array}{l}\text { Salt \& } \\
\text { Pepper noise }\end{array}$ & 21.9674 & 22.7230 & 24.7019 & 11.9235 & 11.2608 & 28.5596 \\
\hline Speckle noise & 22.4662 & 22.9644 & 21.8168 & 14.0948 & 12.9185 & 20.2397 \\
\hline Poisson noise & 28.9512 & 24.1477 & 24.4255 & 16.7472 & 16.3791 & 26.0859 \\
\hline $\begin{array}{l}\text { Uniform } \\
\text { noise }\end{array}$ & 23.5889 & 23.1212 & 24.7775 & 17.7671 & 10.7721 & 28.5887 \\
\hline $\begin{array}{l}\text { Rayleigh } \\
\text { noise }\end{array}$ & 17.9740 & 19.8989 & 23.5495 & 17.9327 & 7.0417 & 26.4316 \\
\hline Erlang noise & 25.2397 & 23.5805 & 24.8731 & 17.7486 & 11.9858 & 28.6750 \\
\hline
\end{tabular}

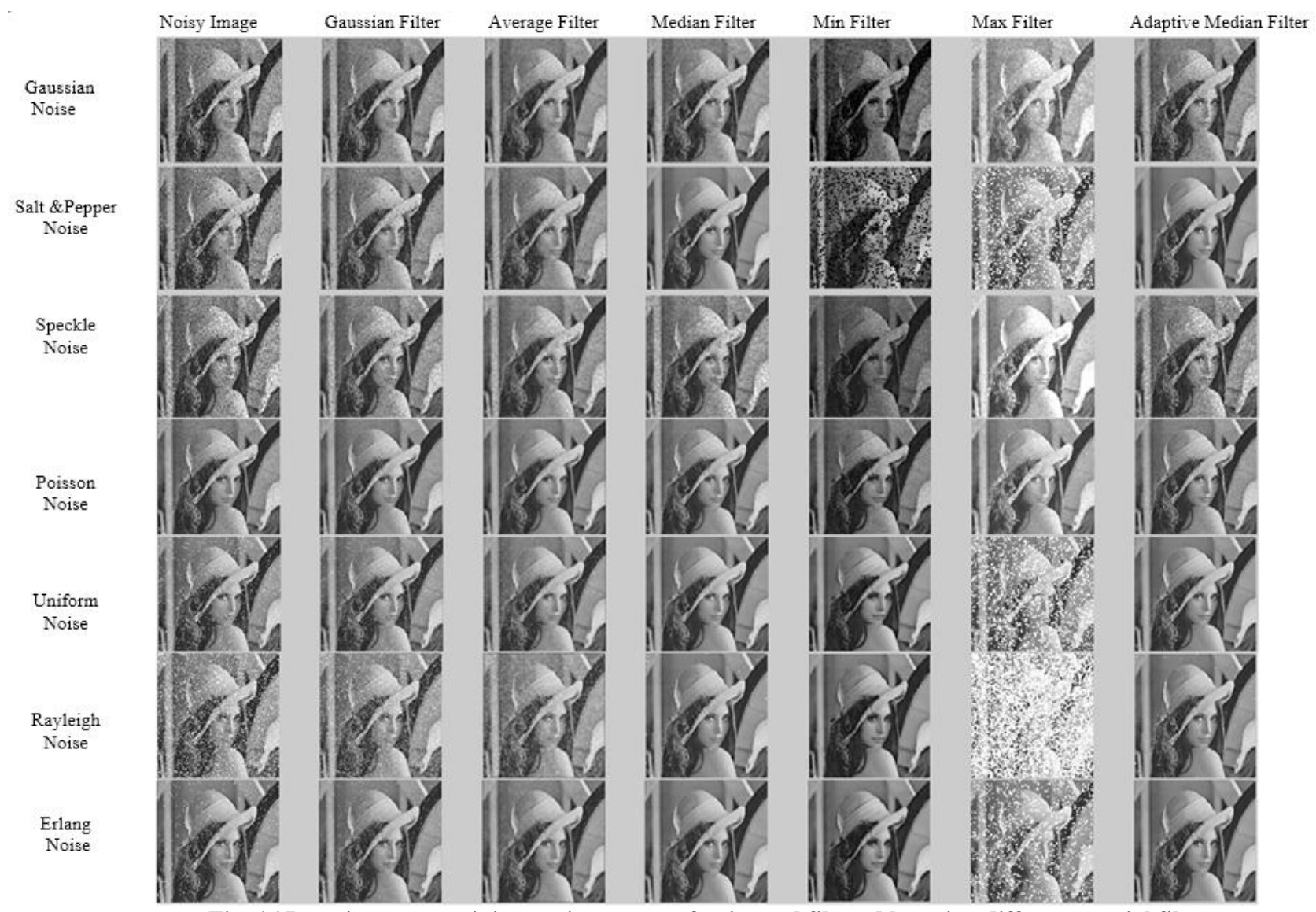

Fig. 16 Lena image containing various types of noise and filtered by using different spatial filters 


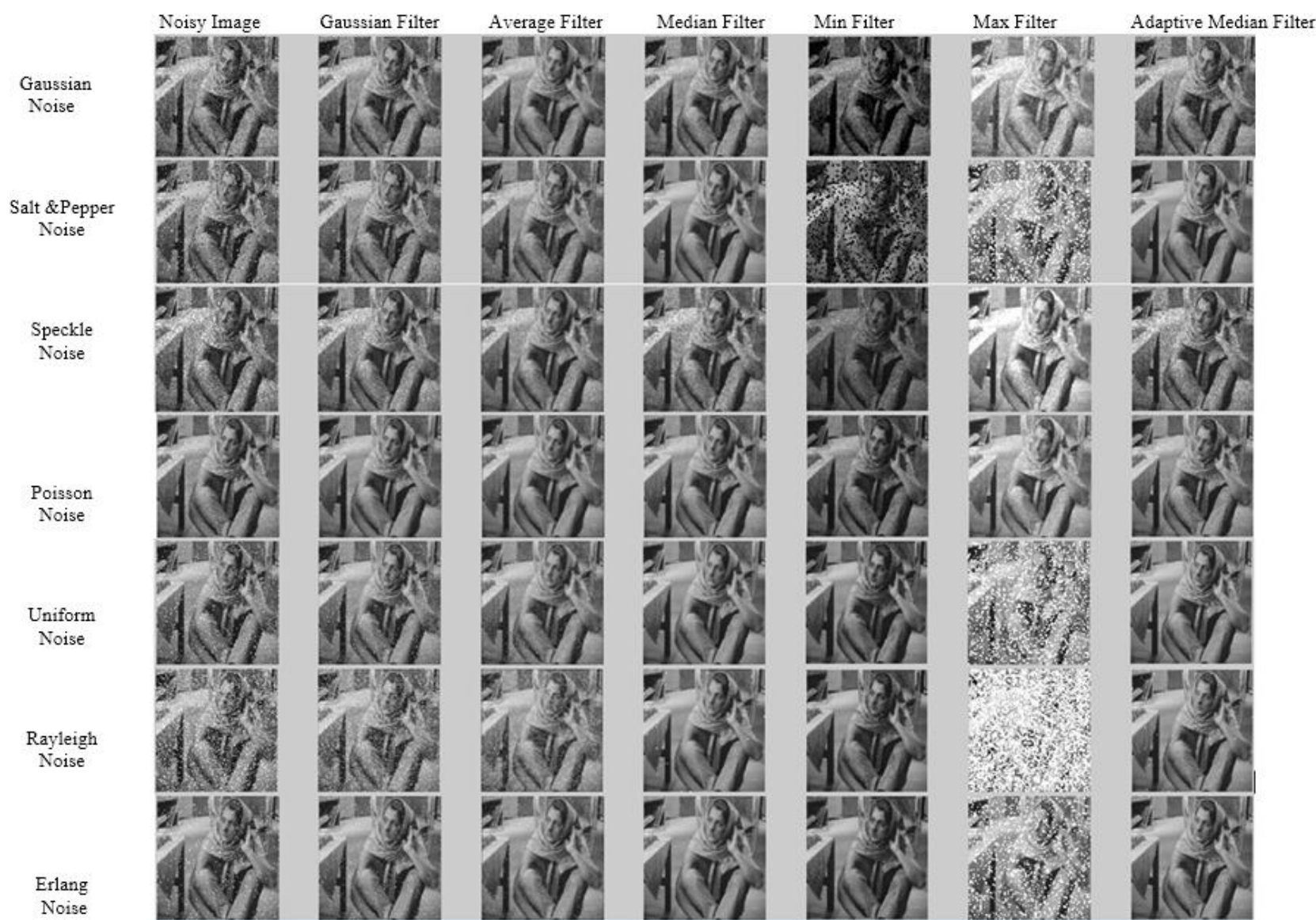

Fig. 17 Barbara image containing various types of noise and filtered by different spatial filters.

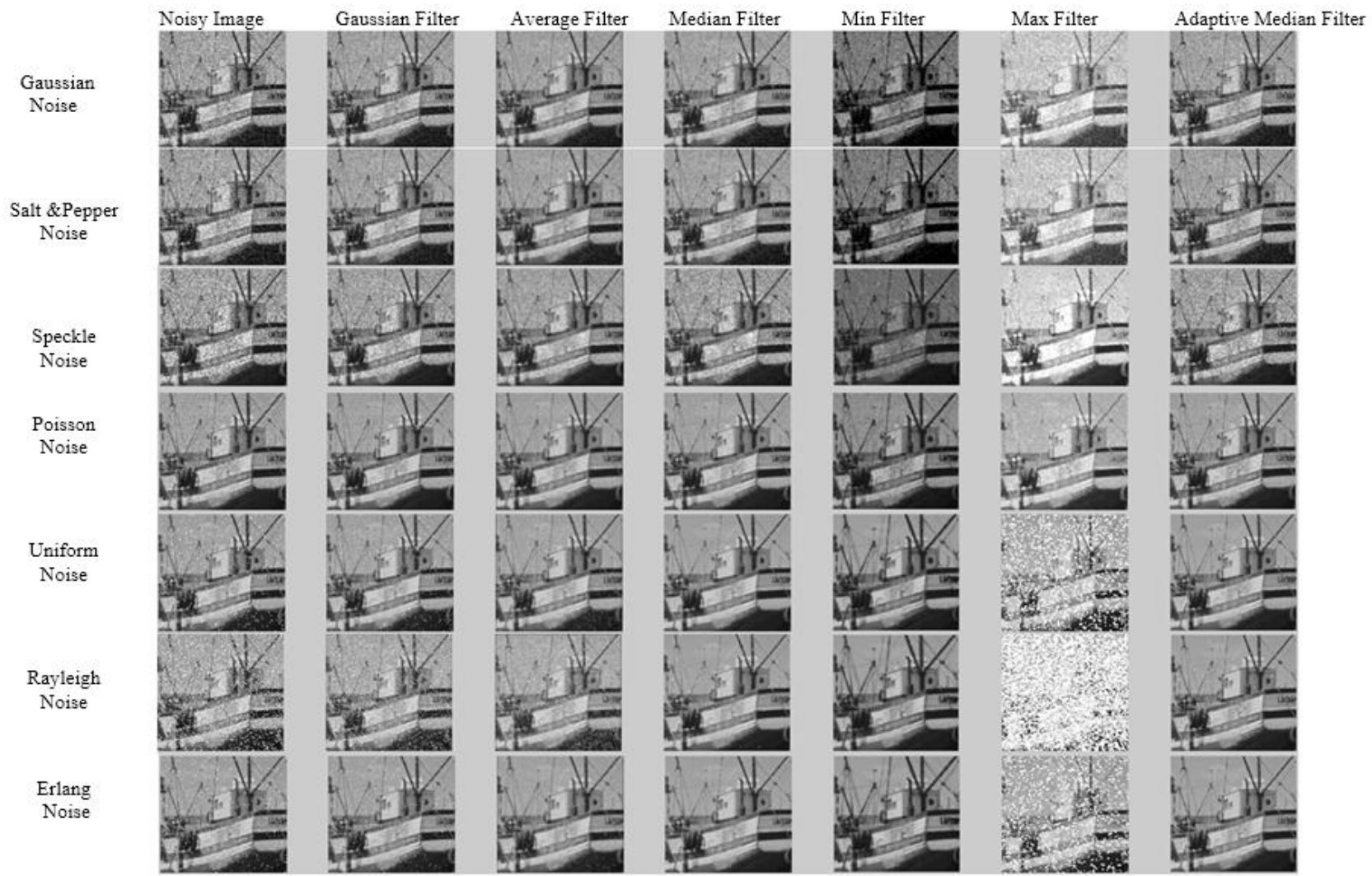

Fig. 18 Boat image containing various types of noise and filtered by different spatial filters 


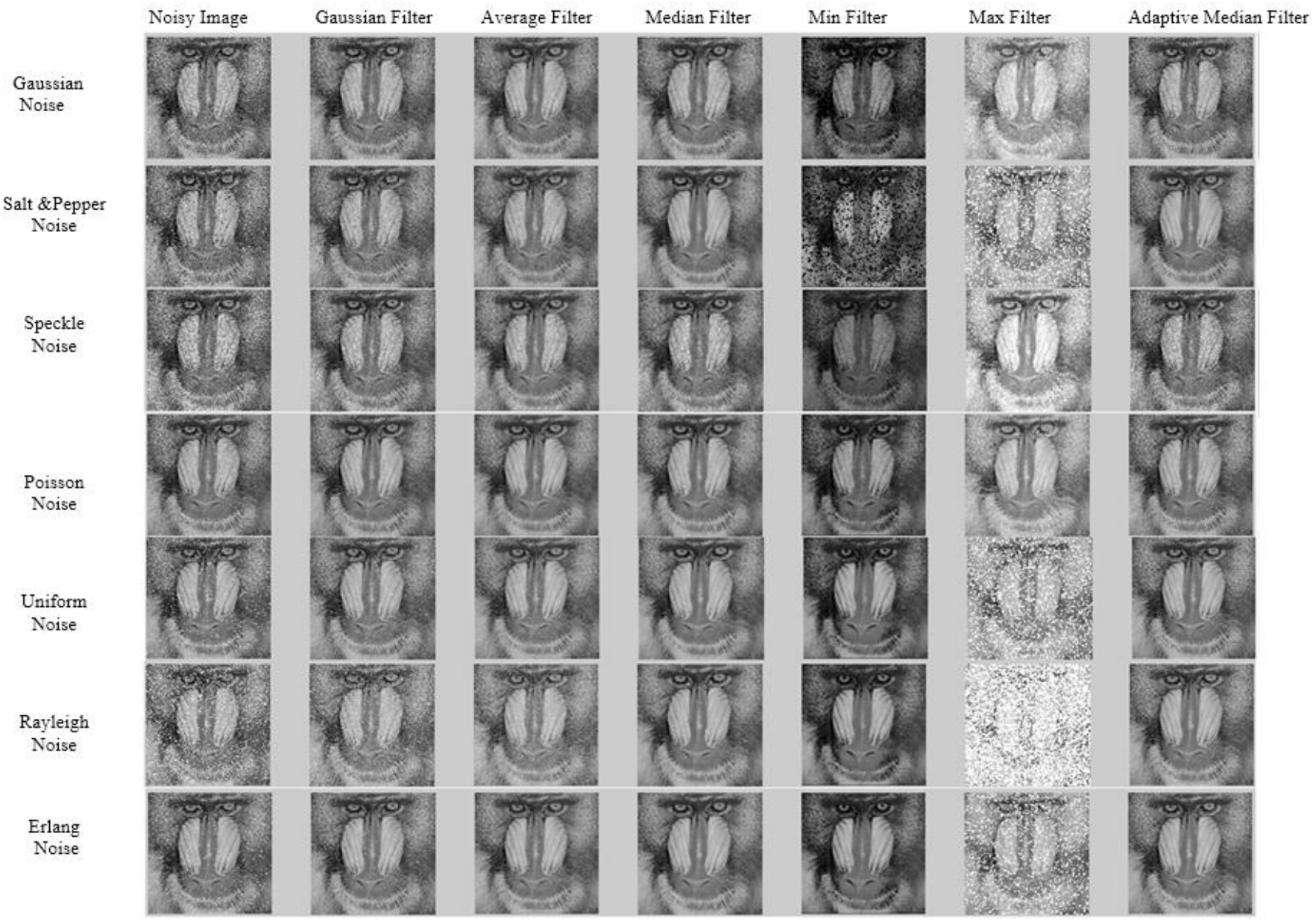

Fig. 19 Baboon image containing various types of noise and filtered by different spatial filters 